http://dx.doi.org/10.35381/r.k.v4i1.457

\title{
Clima organizacional en el desempeño laboral de las instituciones públicas gubernamentales
}

\section{Organizational climate in the work performance of government public institutions}

\author{
Francisca Fernanda Guerrero Chávez \\ amantista fg@hotmail.com \\ Universidad Nacional de Chimborazo, Riobamba \\ Ecuador \\ https://orcid.org/0000-0002-2909-1271 \\ Luis Fernando Neira Altamirano \\ luis.neira@unach.edu.ec \\ Universidad Nacional de Chimborazo, Riobamba \\ Ecuador \\ https://orcid.org/0000-0002-4147-2501 \\ Henry David Vásconez Vásconez \\ hdvasconezv@hotmail.com \\ Escuela Superior Politécnica de Chimborazo, Riobamba \\ Ecuador \\ https://orcid.org/0000-0003-2648-3445
}

Recibido: 10 de agosto de 2019

Aprobado: 30 de agosto de 2019

\section{RESUMEN}

Se tuvo por objetivo determinar la relación de los tipos de clima organizacional en el desempeño laboral de las instituciones públicas gubernamentales. Con un tipo de investigación es correlacional, se fundamentó en un diseño no experimental, transeccional, la población estuvo constituida por un conjunto de 112 sujetos de investigación, que ocupan el cargo de personal administrativo en instituciones públicas gubernamentales de la ciudad de Manta, durante el primer semestre del año 2019. Los climas organizacionales predominantes son el autoritario - explotador y el participativo en grupo, lo cual evidencia que los empleados actúan favorablemente cuando se generan este tipo de clima, favoreciéndose en mayor significancia cuando se emplea el 
participativo de grupo, por cuanto permite que puedan generar aportes que son tenidos en cuenta para el cumplimiento de metas institucionales.

Descriptores: Empresa pública; Administración pública; Cambio organizacional; Administración de empresas.

\begin{abstract}
The objective was to determine the relationship of the types of organizational climate in the work performance of government public institutions. With a type of research is correlational, it was based on a non-experimental, transectional design, the population was constituted by a set of 112 research subjects, who occupy the position of administrative staff in government public institutions of the city of Manta, during the first semester of the year 2019. The predominant organizational climates are the authoritarian - exploitative and participatory - in group, which evidences that the employees act favorably when this type of climate is generated, favoring in greater significance when the group participative is used, because it allows them to generate contributions that are taken into account for the fulfillment of institutional goals.
\end{abstract}

Descriptors: Public enterprises; Public administration; Organizational change; Business management.

\title{
INTRODUCCIÓN
}

El funcionamiento eficaz de las organizaciones requiere de innovación constante en sus planteamientos operativos y en sus principios. Se debe pensar en buscar en el interior de cada organización soluciones que permitan mejorar el trabajo que se realiza para responder de forma favorable a las necesidades que le presenta su contexto interno y externo.

Las organizaciones tienen un propósito, estructura y una colectividad de personas, conformadas por un grupo de elementos interrelacionados entre sí, tales como: estructura organizacional, procesos que se dan dentro de ellas y conducta de los grupos e individuos. Caligiore y Díaz (2003) consideran que "La interacción de estos componentes producen patrones de relación variadas y específicas que encajan en lo que se ha denominado Clima Organizacional" (p. 645). Ante esta situación conviene estudiar el 
clima organizacional, puesto que son diversos los factores que lo determinan, provocando una percepción positiva o negativa en sus integrantes.

Cabe señalar que estas percepciones se traducen en actitudes: positivas en el caso de que la percepción sea favorable; si la percepción es negativa habrá poco compromiso hacia los objetivos de la organización. Marchant, (2005) señala que el clima organizacional hace referencia al ambiente psicosocial en el que se desenvuelven los miembros de una organización, dice relación con el trabajo cotidiano y las metas estratégicas, aprovechando la oportunidad de utilizar una variable no económica para impactar sobre los resultados de la empresa o institución.

De tal manera que el ambiente donde una persona desempeña su trabajo diariamente, el trato que un jefe puede tener con sus subordinados, la relación entre el personal de la empresa e incluso la relación con proveedores y clientes, estos elementos van conformando lo que se denomina Clima Organizacional. Rodríguez (2005), considera que "el clima organizacional es la percepción que los miembros de una organización tienen de las características más inmediatas que les son significativas, que la describen y diferencian de otras organizaciones. Estas percepciones influyen en el comportamiento organizacional "(p.85).

El clima es una variable que media entre la estructura, procesos, metas y objetivos de la empresa, por un lado, y las personas, sus actitudes, comportamiento y desempeño en el trabajo, por otro, se construye a partir de factores extra-organización (macroeconómicos, sociales,) e intra-organización (estructurales, comerciales, individuales y psicosociales). Su poderoso influjo sobre la motivación, el compromiso, la creatividad y el desempeño de las personas y los equipos de trabajo, lo convierten en una herramienta estratégica fundamental para la gestión del recurso humano y el desarrollo organizacional en la empresa contemporánea.

Por lo antes expuesto, el clima organizacional constituye uno de los factores determinantes de los procesos organizativos, de gestión, cambio e innovación. Adquiere relevancia por su repercusión inmediata, tanto en los procesos como en los resultados, lo cual incide directamente en la calidad del propio sistema y su desarrollo. Los cambios 
y las innovaciones en gestión son percibidos de un modo particular por las personas en la organización; ellos se verán afectados y a su vez afectando al clima contingente al proceso de cambio. Un buen clima favorece la actitud de la gente para enfrentar situaciones de incertidumbre, minimizando las implicancias negativas, propias del proceso de cambio, sobre la eficiencia organizacional.

De acuerdo con Marchant (2005). Las organizaciones debieran trabajar en favorecer el desarrollo de un clima organizacional adecuado al trabajo cotidiano y las metas estratégicas, aprovechando la oportunidad de utilizar una variable no económica para impactar sobre los resultados de la empresa o institución. Según Marchant (2005), mientras más satisfactoria sea la percepción que las personas tienen del clima laboral en su empresa, mayor será el porcentaje de comportamientos funcionales que ellos manifiesten hacia la organización y mientras menos satisfactorio sea el clima, el porcentaje de comportamientos funcionales hacia la empresa es menor. Los esfuerzos que haga la empresa por mejorar ciertos atributos del clima organizacional deben retroalimentarse con la percepción que de ellos tienen las personas.

Estas mejoras, mientras sean percibidas como tales, serían el antecedente para que los funcionarios aumenten la proporción de su comportamiento laboral en dirección con los objetivos organizacionales. De igual manera, Goncalves (2000) señala que el conocimiento del Clima Organizacional proporciona retroinformación acerca de los procesos que determinan los comportamientos organizacionales, permitiendo además, introducir cambios planificados tanto en las actitudes y conductas de los miembros, como en la estructura organizacional o en uno o más de los subsistemas que la componen.

La importancia de esta información, se basa en que el Clima Organizacional influye en el comportamiento manifiesto de los miembros, a través de percepciones estabilizadas que filtran la realidad y condicionan los niveles de motivación laboral y rendimiento profesional entre otros. Estudios sistemáticos del clima organizacional permiten efectuar intervenciones certeras tanto a nivel de diseño o rediseño de estructuras organizacionales, planificación estratégica, cambios en el entorno organizacional interno, gestión de programas motivacionales, gestión de desempeño, mejora de sistemas de 
comunicación interna y externa, mejora de procesos productivos, mejora en los sistemas de retribuciones, entre otros.

Cabe destacar que las organizaciones están pasando por una transformación fundamental en todo el mundo, dejando de lado la idea de las organizaciones tradicionales, rígidas, requiriéndose de organizaciones participativas, más planas y con menos niveles jerárquicos, en donde se produzca un mayor acercamiento de todos lo que la integran, con una participación mucho más activa de todo el equipo.

Es importante señalar que para mantener activo los escenarios en donde operan las empresas, sean estas grandes, pequeñas o microempresas, requieren de una gerencia dinámica, innovadora, creativa, capaz de integrar de forma productiva todos los recursos que su organización tiene y sobre todo estar atento de cómo se manifiestan los cambios para mantenerse en los mercados.

En este sentido, la gerencia debe estar atenta al rol que desempeña en pro del factor humano, que además de la remuneración, es necesario atender a las necesidades de participación en la toma de decisiones, saber utilizar, eficientemente todo el legado de conocimiento que debe centrarse en el actual comportamiento del hombre en las organizaciones. Donde no debe descuidar el clima de trabajo, puesto como se sabe, constituye la personalidad de una organización, en el sentido que este está formado por una magnitud de dimensiones que componen su configuración.

El clima organizacional es percibido directa o indirectamente por los trabajadores que se desempeñan en ese medio ambiente, al sentir que la comunicación es abierta y positiva, al sentir que hay entendimiento entre todos los integrantes, no cabe la menor duda que estos se sentirán motivados y satisfechos con su trabajo, lo que impacta positivamente en el desempeño.

Así mismo, un clima organizacional positivo estimula una visión del mundo y la cooperación mutua. Es el ambiente psicológico que promueve la valoración del otro, el respeto y apoyo mutuo, la compensación recíproca, la ayuda desinteresada, la comunicación positiva, los acuerdos, el trabajo en equipo, el aprendizaje conjunto, el 
sentimiento de pertenencia, por cuanto es de interés de los empleados, que su ambiente de trabajo les permita el bienestar personal y les facilite el hacer una buena labor.

Un ambiente físico cómodo y un adecuado diseño del lugar permitirán un mejor desempeño y favorecerá la satisfacción del empleado. En este sentido Stogdill, Good, \& Day (1962), consideran que "el desempeño tiene relación con la satisfacción laboral siempre que exista un reforzamiento en las expectativas del rendimiento". (p.12). Por lo antes expuesto las instituciones públicas deben ser más eficientes en todas las áreas que la integran y para ello se hace necesario que los recursos con los que cuenta se aprovechen eficientemente, teniendo en cuenta su escasez. Optimizar los recursos es una tarea difícil porque las organizaciones son complejas, sobre todo por el hecho de que trabajan mucho con el factor humano, y este tiene innumerables intereses y distintas formas de pensar y percibir las acciones.

Cabe destacar, que uno de los principales capitales que tiene cualquier institución sea público o privado es su talento humano, de cuyo desempeño depende la efectividad de la organización. De tal manera que los integrantes de una organización son personas que en forma activa perciben e interpretan su ambiente laboral. Desde esta perspectiva el clima organizacional es un proceso de medición perceptual del ambiente laboral que influye en las actitudes y conductas de los miembros de una organización. El conocimiento del clima organizacional proporciona información acerca de las percepciones que determinan los comportamientos de los integrantes de la organización y por consiguiente permite introducir cambios planificados con el objeto de influir en dichas percepciones. Estos cambios se transforman en relevantes en la medida que los integrantes de la organización pueden participar en su definición y por supuesto en la acción de actividades en el marco de un programa de intervención permanente.

En virtud de lo planteado, se hace necesario determinar la relación de los tipos de clima organizacional en el desempeño laboral de las instituciones públicas gubernamentales de la ciudad de Manta, durante el primer semestre del año 2019, con la finalidad de conocer el comportamiento de las variables de estudio en conformidad de generar a futuro planes de intervención para la mejora continua. 


\section{METODOLOGÍA DE LA INVESTIGACIÓN}

El tipo de investigación es correlacional porque permite medir la relación existente entre las variables objeto de estudio. En este sentido, Chávez (2007) afirma que estos "estudios correlaciónales tienen como propósito determinar el grado de relación que hay entre dos variables, detectando hasta qué punto las alteraciones de una, dependen de la otra" ( $p$. 137). Se fundamentó en un diseño no experimental, transeccional. El diseño es no experimental porque no se controlarán las variables que se operarán deliberadamente, sino que sólo se observarán las mismas para describirlas y analizarlas derivando teorías. Sobre esto Hernández, Fernández, Baptista (2014) refieren al diseño no experimental como "el diseño donde trabaja transversal o longitudinalmente" (p. 129).

En este caso la población estuvo constituida por un conjunto de 112 sujetos de investigación, que ocupan el cargo de personal administrativo en instituciones públicas gubernamentales de la ciudad de Manta, durante el primer semestre del año 2019. Sabino (2002) en relación a la población "en su conjunto constituye la suma de todas las unidades y se le da el nombre de universo" (p.82). Se aplicó un instrumento de 25 reactivos en escalamiento de Likert de cuatro alternativas de respuestas.

\section{RESULTADOS}

\section{Correlación autoritario - explotador y desempeño laboral Cuadro 1}

\begin{tabular}{llr|r} 
& & AE & \multicolumn{1}{c}{$\mathrm{DL}$} \\
\hline \multirow{2}{*}{$\mathrm{AE}$} & \multicolumn{1}{l}{ Correlación de Pearson } & 1 & \multicolumn{1}{c}{124} \\
\cline { 2 - 4 } & \multicolumn{1}{l}{ Sig. (bilateral) } & &, 194 \\
\cline { 2 - 4 } & $\mathrm{N}$ & 112 & 112 \\
\hline \multirow{2}{*}{$\mathrm{NL}$} & Correlación de Pearson &, 124 & 1 \\
\cline { 2 - 4 } & Sig. (bilateral) &, 194 & \\
\hline & $\mathrm{N}$ & 112 & 112 \\
\hline
\end{tabular}

El indicador autoritario - explotador y desempeño laboral, tiene una significancia de 
0,194 y una correlación de 0,124, catalogándose en una correlación positiva moderada, lo cual implica que ambas variables interactúan entre sí para beneficio mutuo.

\section{Correlación autoritario-paternalista y desempeño laboral \\ Cuadro 2}

\begin{tabular}{llr|r} 
& & $\mathrm{DL}$ & \multicolumn{1}{c}{ AP } \\
\hline \multirow{2}{*}{$\mathrm{DL}$} & Correlación de Pearson & 1 &,- 067 \\
\cline { 2 - 4 } & Sig. (bilateral) & &, 481 \\
\cline { 2 - 4 } & $\mathrm{N}$ & 112 & 112 \\
\hline \multirow{2}{*}{$\mathrm{AP}$} & Correlación de Pearson &,- 067 & 1 \\
\cline { 2 - 4 } & Sig. (bilateral) &, 481 & \\
\hline $\mathrm{N}$ & 112 & 112 \\
\hline
\end{tabular}

El indicador autoritario - paternalista y desempeño laboral, tiene una significancia de 0,481 y una correlación de - 0,067, catalogándose en una correlación negativa baja, lo cual implica que ambas variables no interactúan entre sí para beneficio mutuo. 
Correlación participativo - consultivo y desempeño laboral Cuadro 3

\begin{tabular}{|c|c|c|c|}
\hline & & PC & DL \\
\hline \multirow[t]{3}{*}{ PC } & Correlación de Pearson & 1 & ,093 \\
\hline & Sig. (bilateral) & & ,331 \\
\hline & $\mathrm{N}$ & 112 & 112 \\
\hline \multirow[t]{3}{*}{ DL } & Correlación de Pearson & ,093 & 1 \\
\hline & Sig. (bilateral) & ,331 & \\
\hline & $\mathrm{N}$ & 112 & 112 \\
\hline
\end{tabular}

El indicador participativo - consultivo y desempeño laboral, tiene una significancia de 0,331 y una correlación de - 0,093, catalogándose en una correlación positiva moderada, lo cual implica que ambas variables interactúan entre sí para beneficio mutuo.

\section{Correlación participativo - en grupo y desempeño laboral Cuadro 4}

\begin{tabular}{|c|c|c|c|}
\hline & & $P G$ & DL \\
\hline \multirow[t]{3}{*}{$P G$} & Correlación de Pearson & 1 &, $392^{* *}$ \\
\hline & Sig. (bilateral) & &, 000 \\
\hline & $\mathrm{N}$ & 112 & 112 \\
\hline \multirow[t]{3}{*}{$\mathrm{DL}$} & Correlación de Pearson &, $392^{* *}$ & 1 \\
\hline & Sig. (bilateral) &, 000 & \\
\hline & $\mathrm{N}$ & 112 & 112 \\
\hline
\end{tabular}

**. La correlación es significativa en el nivel 0,01 (bilateral).

El indicador participativo - en grupo y desempeño laboral, tiene una significancia de 
0,000 y una correlación de 0,392 catalogándose en una correlación positiva alta, lo cual implica que ambas variables interactúan entre sí para beneficio mutuo.

\section{DISCUSIÓN}

En cuanto al clima tipo autoritario - explotador, se tiene que incide en el desempeño laboral de la población objeto de estudio, Brunet (2002), indica que este tipo de clima se caracteriza porque:

La dirección no tiene confianza en sus empleados, la mayor parte de las decisiones y de los objetivos se toman en la cima de la organización y se distribuyen según una función puramente descendente. Los empleados tienen que trabajar dentro de una atmósfera de miedo, de castigos, de amenazas, ocasionalmente de recompensas, y la satisfacción de las necesidades permanece en los niveles psicológicos y de seguridad, este tipo de clima presenta un ambiente estable y aleatorio en el que las comunicaciones de la dirección con sus empleados no existe más que en forma de ordenes e instrucciones específicas.

Se tiene que la población de estudio se caracteriza por tener un nivel de confianza generado al cumplir con las órdenes del jefe, lo cual les brinda estabilidad en el puesto de trabajo por cuanto esto implica que existe una remuneración garantizada, enfoque desde el cual el empleado planifica su estilo de vida, siendo importante mantener su puesto de trabajo.

En la medición autoritario-paternalista y desempeño laboral, al existir una correlación negativa, se plantea un distanciamiento con el planteamiento teórico de Brunet (2002), dado que no se evidencia confianza condescendiente entre el jefe y subordinados, los empleados escasamente toman decisiones e iniciativas para ejercer su trabajo de modo efectivo, quedando a la toma de decisiones en los niveles alto de la institución.

Al existir una correlación positiva en la medición participativo - consultivo y desempeño laboral, se evidencia que los jefes realizan consultas a los subordinados, sin que esto signifique que se tenga en consideración los planteamientos de los empleados, se denota la existencia de recompensas para la realización de trabajos específicos, lo cual concuerda con los planteamientos de Brunet (2002). 
En cuanto al indicador participativo - en grupo y desempeño laboral, se desarrolla una correlación significativa, lo cual permite comprender que cuando se trabaja en consulta del equipo de trabajo, se brindan oportunidades de participación, donde los empleados puedan contribuir de modo efectivo en la puesta en marcha de planes de acción, se genera un clima de cordialidad y confianza, lo cual concuerda con Brunet (2002).

\section{CONCLUSIONES}

Los climas organizacionales predominantes son el autoritario - explotador y el participativo - en grupo, lo cual evidencia que los empleados actúan favorablemente cuando se generan este tipo de clima, favoreciéndose en mayor significancia cuando se emplea el participativo de grupo, por cuanto permite que puedan generar aportes que son tenidos en cuenta para el cumplimiento de metas institucionales.

La tendencia de las organizaciones públicas y privadas es de brindar mayor participación a sus empleados, fomentar el trabajo en equipo y cooperativo para la generación de una sinergia que permita el logro de metas, objetivos, en función del aporte brindando por cada miembro de la institución, de ese modo, se trabaja en el enfoque de estimular las potencialidades de la persona, promoviéndose la mejora continua.

Sin embargo, en las instituciones públicas, suele emplearse la visión autoritaria con la finalidad de coaccionar para el cumplimiento de metas, siendo esta medida alejada de la tendencia empresarial que propone el trabajo cooperativo, aunque el autoritario pueda ser efectivo en determinadas ocasiones, a la larga podría resultar en detrimento de la propia organización, debido que se genera un clima basado en la generación de órdenes para el cumplimiento de acciones por parte del empleado, minimizándose cualidades como la iniciativa, proactividad, compromiso, sinergia, para el desarrollo de un clima organizacional que favorezca al trabajo en equipo de modo efectivo y asertivo, siendo necesario tener en cuenta a Borrero (2019), al indicar:

Las organizaciones exitosas, descubren en el valor y las habilidades de las personas que están en ella, su principal ventaja competitiva, por ello, tratan a sus miembros como socios de la empresa y no como simples empleados, trabajadores o recursos productivos de la organización (p. 295). 
Esta opción de empresa debe ser manejada por lo público, con la finalidad de generar relaciones de trabajo en conformidad a la generación de un clima que permita promover desde las múltiples competencias de los miembros de la organización, trabajar no solo en metas y objetivos, sino en base a la conformación de equipos de trabajos que funcionen en un marco de cordialidad, respeto, logrando sinergia como parte de una gestión organizacional efectiva y exitosa, aunado a lo planteado por Aldana, Salón \& Guzmán (2019), quienes plantean la necesidad de contar con una visión sistémica en la organización que permita contribuir en la fusión de las potencialidades del talento humano para el logro de una gestión eficiente basada en el respeto mutuo de las interrelaciones generadas en la empresa.

\section{REFERENCIAS CONSULTADAS}

1. Aldana, J., Salón, M., \& Guzmán, N. (2019). Liderazgo sistémico en las competencias gerenciales docentes universitarias. CIENCIAMATRIA, 5(8), 50-74. Recuperado a partir de http://cienciamatriarevista.org.ve/index.php/cm/article/view/87

2. Borrero, R. (2019). Procesos de gestión del talento humano en el sector educativo gerencial de Colombia. Revista Arbitrada Interdisciplinaria Koinonía, 4(7), 293-307. doi:http://dx.doi.org/10.35381/r.k.v4i7.206

3. Brunet L. (2002). El Clima de Trabajo en las Organizaciones: Definiciones, diagnóstico y consecuencias. México: Editorial Trillas.

4. Chávez, N. (2007). Introducción a la Investigación. Educativa. Ediciones Grafica, C.A. Maracaibo - Venezuela.

5. Caligiore, C. \& Díaz T. (2003). Clima Organizacional y Desempeño de los Docentes en la ULA. Estudio de un caso. Revista Venezolana de Gerencia (RVG). Año 8 №24 Universidad del Zulia (LUZ) Maracaibo. Venezuela. pp. 644 - 656.

6. Goncalves, A. (2000). Fundamentos del Clima Organizacional. Sociedad Latinoamericana para la Calidad. (SLC). 
7. Hernández, R., Fernández, C., Baptista, P. (2014). Metodología de la Investigación. Editorial Mc - Graw - Hill Interamericana. Sexta edición.

8. Marchant, L. (2005) Actualizaciones para el desarrollo organizacional primer seminario Viña del Mar. Chile • Marchant, L.; Prieto, A. (2005) "Cuestionario CCO (Copyright 2005, N 149.282. Viña del Mar. Chile

9. Rodríguez, L. (2005). Diagnóstico del clima organizacional. Recuperado de www.kpmg. com.pa/ Publicación /.../ clima organizacional. Consultado 15/4/2010-

10. Stogdill, R., Good, O. \& Day, D. (1962). New LeaderBehavior Description Subscales. Journal of Psy-chology, 54, 259-269.

\section{REFERENCES CONSULTED}

1. Aldana, J., Salón, M., \& Guzmán, N. (2019). Systemic leadership in university teaching management skills. SCIENCE, 5 (8), 50-74. Recovered from http://cienciamatriarevista.org.ve/index.php/cm/article/view/87

2. Borrero, R. (2019). Human talent management processes in the management education sector of Colombia. Interdisciplinary Arbitrated Review Koinonía, 4 (7), 293-307. doi: http: //dx.doi.org/10.35381/r.k.v4i7.206

3. Brunet L. (2002). The Climate of Work in Organizations: Definitions, diagnosis and consequences. Mexico: Editorial Trillas.

4. Chavez, N. (2007). Introduction to research. Educational. Ediciones Grafica, C.A. Maracaibo Venezuela.

5. Caligiore, C. \& Díaz T. (2003). Organizational Climate and Teacher Performance at the ULA. Case study Venezuelan Management Magazine (RVG). Year 8 №24 University of Zulia (LUZ) Maracaibo. Venezuela. pp. 644-656.

6. Goncalves, A. (2000). Fundamentals of Organizational Climate. Latin American Society for Quality. (SLC).

7. Hernández, R., Fernández, C., Baptista, P. (2014). Investigation methodology. Editorial Mc - Graw - Interamerican Hill. Sixth edition.

8. Marchant, L. (2005) Updates for the organizational development first seminar Viña del Mar. Chile • Marchant, L;Prieto, A. (2005) "CCO Questionnaire (Copyright 2005, N 149.282. Viña del Mar. Chile. 
9. Rodríguez, L. (2005). Diagnosis of the organizational climate. Recovered from www.kpmg. com.pa/ Publication /.../ organizational climate. Consulted 15/4 / 2010-

10. Stogdill, R., Good, O. \& Day, D. (1962). New LeaderBehavior Description Subscales. Journal of Psy-chology, 54, 259-269.

(C2019 por los autores. Este artículo es de acceso abierto y distribuido según los términos y condiciones de la licencia Creative Commons Atribución-NoComercial-Compartirlgual 4.0 Internacional (CC BY-NC-SA 4.0)

(https://creativecommons.org/licenses/by-nc-sa/4.0/). 